\title{
Fluorophotometric measurement of the precorneal residence time of topically applied hyaluronic acid
}

\author{
H Mochizuki, ${ }^{1}$ M Yamada, ${ }^{1}$ S Hato, ${ }^{1}$ T Nishida ${ }^{2}$
}

${ }^{1}$ Division for Vision Research, National Institute of Sensory Organs, National Tokyo Medical Center, Tokyo, Japan;

${ }^{2}$ Department of Ophthalmology, Yamaguchi University Graduate School of Medicine, Yamaguchi, Japan

Correspondence to:

M Yamada, Division for Vision Research, National Institute of Sensory Organs, National Tokyo Medical Center, 2-5-1

Higashigaoka, Meguro, Tokyo 152-8902, Japan;

yamadamasakazu@kankakuki. go.jp

Accepted 6 June 2007

\section{ABSTRACT}

Purpose: This study was performed to separately assess the aqueous flow applied with hyaluronic acid, and the behaviour of hyaluronic acid itself on the ocular surface. Methods: Two different fluorescent dyes, fluorescein sodium dissolved in $0.1 \%$ hyaluronic acid (HA) solution and $0.1 \%$ fluorescein conjugated with hyaluronic acid (FHA) dissolved in saline, were used. A volume of $20 \mu \mathrm{l}$ of tested solution was applied to the eye of 10 healthy volunteers. Fluorescein sodium dissolved in saline served as a control. The fluorescent intensity of the precorneal tear film was measured at the central cornea every minute for $10 \mathrm{~min}$. The turnover rate was calculated using the equation that plots fluorescent intensity against time in a semilog plot and expressed as \%/min.

Results: Turnover rates of topically applied 0.1\% F-HA, $0.1 \% \mathrm{HA}$ and saline were 8.1 (SD 3.6)\%/min, 21.6 (2.8)\%/ $\mathrm{min}$, and $31.0(3.7) \% / \mathrm{min}$, respectively. The turnover rate of $\mathrm{F}-\mathrm{HA}$ was significantly lower than those of $\mathrm{HA}$ and saline $(p=0.00012$ and $p=0.00000022$, respectively; Mann-Whitney test). The turnover rate of HA was significantly lower than that of saline $(p=0.00001$; Mann-Whitney test).

Conclusion: Our results indicate that the bulk aqueous flow applied with $\mathrm{HA}$ and the turnover of $\mathrm{HA}$ itself are different. HA molecules may adhere to the ocular surface by surface-chemical and/or biochemical properties. The long retention time of HA on the ocular surface may explain the mechanism in which hyaluronic acid has been shown to enhance tear film stability for a few hours.

Dry eye is a common condition, affecting approximately $10-20 \%$ of the adult population. ${ }^{1}$ The clinical consequences of dry eye may include symptomatic irritation, superficial punctuate keratopathy, corneal erosions and possibly visual acuity problems. ${ }^{2}$ Dry eye is considered to be a disorder of the tear film due to tear deficiency or excessive evaporation, which causes damage to the interpalpebral ocular surface and is associated with symptoms of ocular discomfort. ${ }^{3}$

A variety of treatment modalities have been used for the treatment of dry eye. The majority of these fall into the category of tear substitutes or replacements. ${ }^{4}$ Artificial tears, the most frequently used modality for the treatment of dry eye, may be effective in relieving symptoms in mild dry eye by replenishing deficient tear volume. However, in moderate and severe cases of dry eye, artificial tears alone are not enough to relieve the symptoms nor to improve superficial punctuate keratopathy. ${ }^{4}$

Since preliminary reports in the early $1980 \mathrm{~s}^{, 5-7}$ several studies have reported that hyaluronic acid is able to improve the symptoms, signs and ocular surface damage associated with dry eye syndrome..$^{8-11}$ Hyaluronic acid is a glycosaminoglycan with a viscoelastic rheology. Its relatively high viscosity is believed to improve tear-film stability and to reduce washout from the ocular surface. ${ }^{12}$ Hyaluronic acid enhances water retention on the corneal surface, and probably increases corneal wettability ${ }^{13}$ In addition, hyaluronic acid promotes migration of corneal epithelial cells and accelerates the healing of corneal epithelial defects. ${ }^{14-16}$ Hyaluronic acid has thus become an important treatment modality for dry eye.

Conflicting results, however, have been obtained regarding the duration of the action of hyaluronic acid on the ocular surface. The residence time of topically applied hyaluronic acid assessed by the tear meniscus height and water evaporation rate from the ocular surface was less than $10 \mathrm{~min}$, although this was significantly longer than that of phosphate-buffered saline. ${ }^{17} 18$

Using an assessment of the tear-film breakup time, however, hyaluronic acid has been shown to enhance tear-film stability for more than a few hours. ${ }^{19-21}$ These observations suggest that hyaluronic acid remains on the ocular surface independent of the bulk aqueous flow.

In order to test this hypothesis, we used two different fluorescent dyes, fluorescein sodium dissolved in hyaluronic acid solution and fluorescein conjugated with hyaluronic acid dissolved in saline, in the current study. The former is a wellestablished dye used to assess the bulk aqueous flow, and the latter dye is used as a tracer to determine the behaviour of hyaluronic acid on the ocular surface. Although the residence time of topically applied hyaluronic acid has been investigated using ${ }^{99} \mathrm{Tc}^{\mathrm{m}}$ as a tracer, ${ }^{22-24}$ we believe that this study is the first report to measure the residence time using a tracer that is associated with hyaluronic acid on the ocular surface.

\section{SUBJECTS AND METHODS}

\section{Fluorescent dye and fluorophotometer}

Fluorescein hyaluronic acid (F-HA, Mw; 800000 $\mathrm{Da}$ ) was purchased from Sigma-Aldrich (St. Louis, $\mathrm{MO}$ ). F-HA, fluorescein conjugated with hyaluronic acid, was dissolved in a phosphate-buffered saline as $0.1 \%$ solution and used as a tracer of hyaluronic acid. Fluorescein sodium $(0.001 \%$; Sigma-Aldrich) and $0.1 \%$ hyaluronic acid (Mw; $800000 \mathrm{Da}$, Sigma-Aldrich) dissolved in a phosphate-buffered saline was used as a tracer of the bulk aqueous flow in the presence of hayaluronic acid. Fluorescein sodium $(0.001 \%)$ in a phosphatebuffered saline was used as a tracer of the bulk aqueous flow. online under the BMJ Journals unlocked scheme, see http:// bjo.bmj.com/info/unlocked.dtl 
A commercial slit-lamp fluorophotometer (Anterior Fluorometer FL-500, Kowa Co., Tokyo) was used. The illuminating light was focused as a 2-mm diameter circle on the surface of the cornea. The emitted light passed through a band-interference filter centred on $565 \mathrm{~nm}$ (half bandwidth $25 \mathrm{~nm}$ ) and was directed to a photomultiplier tube with the band-interference filter centred on wavelengths $490 \mathrm{~nm}$ (half bandwidth $30 \mathrm{~nm}$ ).

F-HA solution (0.5\%) was diluted in a phosphate-buffered saline to produce sets of standards ranging from $0.001 \%$ to $0.5 \%$ in concentration for the calibration. A cuvette was constructed by gluing together two microscope slides and two cover glasses. The cover glasses were sandwiched by two microscope slides in order to provide space for the fluid layer to be $12-15 \mu \mathrm{m}$ thick. A fresh one was made for each solution. Ten microlitres of calibrating fluids, containing $0.001-0.5 \%$ F-HA, was placed into a cuvette. The fluorescent intensity was measured by a slit-lamp fluorophotometer. The interaction of F-HA with the proteins was also tested using a phosphate-buffered saline containing $1 \%$ fetal bovine serum.

\section{Measurement of residence time}

Ten healthy volunteers (five male and five female) aged 2744 years (33.8 (SD 6.8) years old, mean (SD)), who had no history of eye disease, except for refractive errors, were enrolled in this study. The principles of the World Medical Association Declaration of Helsinki were followed. The subjects received a full explanation of the procedures, and provided their informed consent for participation prior to the experiment. The protocol was approved by our institutional review board, and all subjects provided their written informed consent.

In our experiments, the subjects were seated in front of the fluorophotometer. The instrument was focused on the central cornea, and the background fluorescent intensity was measured. A volume of $20 \mu \mathrm{l}$ of tested solution was applied to the eye with an Eppendorf micropipette without making contact. The subjects were then instructed to blink several times to ensure the mixing of the dye. The fluorescent intensity of the precorneal tear film was measured at the central cornea every minute for $10 \mathrm{~min}$. Repeated measurements on different days were made in some subjects to evaluate the repeatability of the test.

The turnover rate is given by the following equation, which plots fluorescent intensity against time in a semilog plot:

$$
\mathrm{F}=\mathrm{F}_{0} \exp (-\mathrm{kt})
$$

where $\mathrm{F}$ is the fluorescent intensity at time $(t) ; F_{0}$ is the fluorescent intensity at time zero; $\mathrm{k}$ is the turnover rate; and $\mathrm{t}$ is the time in minutes. ${ }^{25}$ The turnover rate was calculated using the equation and expressed as $\% / \mathrm{min}$. The regression fit of the $\log$ of the fluorescent intensity was recorded as the regression coefficient.

In all cases of $0.1 \% \mathrm{~F}-\mathrm{HA}$ and $0.1 \%$ hyaluronic acid, this regression became a straight line. In cases of saline, however, this regression sometimes showed a biphasic response: an initial faster and a subsequent lower turnover rate. When the turnover rate of saline became biphasic, the subsequent lower turnover rate was used as the flow rate of saline. ${ }^{25}$

All results are presented as the mean \pm 1 standard deviation (SD). Statistical significance was calculated by comparing results using the Mann-Whitney test. A value of $p<0.05$ was considered to indicate statistical significance.

\section{RESULTS}

\section{Calibration of F-HA}

The calibration of the fluorescent intensities against the concentrations of $0.001-0.5 \% \mathrm{~F}-\mathrm{HA}$ is shown in fig 1 . The relationship between the fluorescent intensities and the concentrations of F-HA was linear $\left(r^{2}=0.995\right)$. The data generated by this method were consistent and reproducible. The fluorescent intensities of F-HA were unaffected by the presence of $1 \%$ fetal bovine serum (data not shown).

\section{Turnover-rate measurements}

A typical result of turnover-rate measurements obtained from one subject is shown in fig 2. In the presented case, the fluorescent intensities of $0.1 \%$ F-HA decayed with time at a flow rate of $7.6 \% / \mathrm{min}$, which was lower than those of fluorescein sodium in $0.1 \%$ hyaluronic acid $(19.4 \% / \mathrm{min})$ and in saline $(28.1 \% / \mathrm{min})$.

The turnover rates of topically applied F-HA, hyaluronic acid and saline obtained from 10 subjects were $8.1(3.6) \% / \mathrm{min}, 21.6$ $(2.8) \% / \mathrm{min}$, and $31.0(3.7) \% / \mathrm{min}$, respectively (table 1$)$. The turnover rate of F-HA was significantly lower than those of hyaluronic acid and saline $(p=0.00012$, and $p=0.00000022$, respectively; Mann-Whitney test). The turnover rate of F-HA was significantly lower than that of saline ( $p=0.00001$; MannWhitney test).

\section{DISCUSSION}

In the current study, we used two different fluorescent dyes, fluorescein sodium dissolved in hyaluronic acid solution and FHA solution, to separately assess the aqueous flow applied with hyaluronic acid, and the behaviour of hyaluronic acid itself on the ocular surface. Our results indicate that there are two different aspects of the duration of topically applied hyaluronic acid.

Hyaluronic acid has a high-molecular-weight, naturally occurring glycosaminoglycan. Its relatively high viscosity is believed to reduce washout from the ocular surface. ${ }^{13}$ The residence time of topically applied hyaluronic acid has previously been investigated by using gamma scintigraphic methods. ${ }^{22-24}$ Snibson and associates ${ }^{22}{ }^{24}$ reported that hyaluronic acid had prolonged ocular residence times in comparison

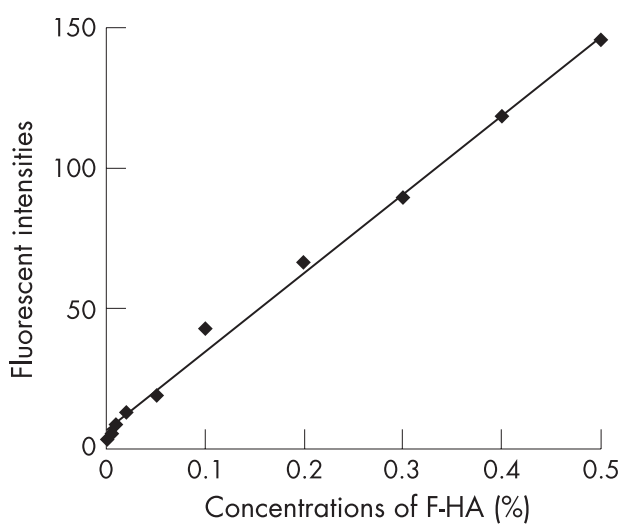

Figure 1 Calibration of the fluorescent intensities against the concentrations of flurorescein hyaluronic acid (F-HA). The relationship between the fluorescent intensities and the concentrations of F-HA was linear $\left(r^{2}=0.995\right)$. 
Table 1 Turnover rates of topically applied $0.1 \%$ fluorescein hyaluronic acid (F-HA), $0.1 \%$ hyaluronic acid and saline obtained from 10 subjects

\begin{tabular}{|c|c|c|c|}
\hline \multirow[b]{2}{*}{ Subject no. } & \multicolumn{3}{|c|}{ Turnover rate $(\% / \mathrm{min})$} \\
\hline & F-HA & Hyaluronic acid & Saline \\
\hline 1 & 7.6 & 19.4 & 28.1 \\
\hline 2 & 12.2 & 22.6 & 31.0 \\
\hline 4 & 9.5 & 20.4 & 33.2 \\
\hline 5 & 4.6 & 21.4 & 33.4 \\
\hline 6 & 8.6 & 22.2 & 37.1 \\
\hline 7 & 15.2 & 24.2 & 29.6 \\
\hline 10 & 7.1 & 21.3 & 33.9 \\
\hline Mean (SD) & $8.1(3.6)$ & $21.7(2.8)$ & $31.0(3.7)$ \\
\hline
\end{tabular}

The turnover rate of F-HA was significantly lower than those of hyarulonic acid and saline ( $p=0.00012$ and $p=0.00000022$, respectively; Mann-Whitney test).

with a buffered saline solution, a solution containing polyvinyl alcohol or hydroxypropylmethylcellulose. In the current study, the turnover rate of the $0.1 \%$ hyaluronic acid solution $(21.6$ $(2.8) \% / \mathrm{min}$ ) was significantly lower than that of the saline $(31.0$ $(3.7) \% / \mathrm{min})$. Our result is considered to be in good accordance with the previous studies using scintigraphic methods. ${ }^{22-24}$ This effect, however, appears to be transient, because $90 \%$ of the hyaluronic acid solution was calculated to be cleared from the ocular surface $10.7 \mathrm{~min}$ after instillation. This result is also consistent with the duration of topically applied hyaluronic acid assessed by the tear meniscus height and water evaporation rate from the ocular surface. ${ }^{17} 18$

The most interesting finding of the current study is that the turnover rate of F-HA (8.1 (3.6)\%/min) was approximately onethird of the $0.1 \%$ hyaluronic acid solution $(21.6(2.8) \% / \mathrm{min})$. This result indicates that the bulk aqueous flow applied with hyaluronic acid and the turnover of hyaluronic acid itself on the ocular surface are different. Besides viscosity, hyaluronic acid molecules may adhere to the ocular surface by surface-chemical and/or biochemical properties, because hyaluronic acid is known to bind with fibronectin and CD44, a cell surface adhesion molecule which has been found on corneal epithelial cells. ${ }^{16}{ }^{26}$ Snibson and associates ${ }^{24}$ made a similar consideration based on their scintigraphic results. They, however, also mentioned the limitation of their methodology and the necessity of tracers that directly associate with hyaluronic acid. We believe that F-HA is a useful tracer to determine the behaviour of topically applied hyaluronic acid. According to our

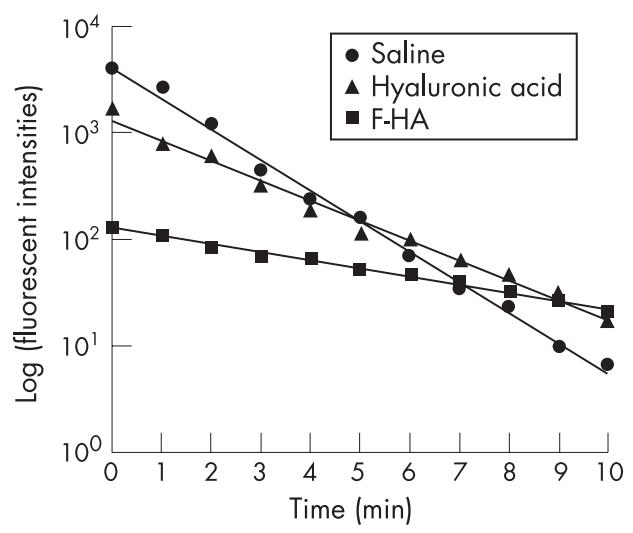

Figure 2 Typical result of the turnover rate measurements obtained from one subject. In the presented case, the fluorescent intensities of $0.1 \%$ flurorescein hyaluronic acid (F-HA) decayed with time at a flow rate of $7.6 \% / \mathrm{min}$, which was lower than those of fluorescein sodium in $0.1 \%$ hyaluronic acid $(19.4 \% / \mathrm{min})$ and in saline $(28.1 \% / \mathrm{min})$. results, the times for $90 \%$ and $99 \%$ of hyaluronic acid to be cleared from the ocular surface were calculated to be $28.8 \mathrm{~min}$ and $57.6 \mathrm{~min}$ after instillation, respectively. The long retention time of hyaluronic acid in the ocular surface may explain the fact that hyaluronic acid has been shown to enhance tear-film stability for more than a few hours. ${ }^{19-21}$

Besides its biological effects on the corneal epithelial cells, hyaluronic acid appears to have two beneficial effects for the treatment of dry eye syndrome. First, it reduces the bulk aqueous flow by its viscosity and increases tear volume for a limited time, as do other viscous agents, such as chondroitin sulfate, polyvinyl alcohol and hydroxypropylmethylcellulose. ${ }^{22}$ Second, hyaluronic acid remains on the ocular surface for a longer time, in order to increase corneal wettability and to retain tear fluid on the corneal surface. ${ }^{12}{ }^{13}$ This effect may be unique for hyaluronic acid, although it should be confirmed by further investigations.

Competing interests: The authors have no proprietary interest in any materials in this manuscript.

\section{REFERENCES}

1. Johnson ME, Murphy PJ. Changes in the tear film and ocular surface from dry eye syndrome. Prog Retin Eye Res 2004;23:449-74.

2. Holy FJ, Lemp MA. Tear physiology and dry eyes. Surv Ophthalmol 1977;22:69-87.

3. Lemp MA. Report of the National Eye Institute/Industry workshop on clinical trials in dry eyes. CLAO J 1995;21:221-32.

4. Nelson JD, Farris RL. Sodium hyaluronate and polyvinyl alcohol artificial tear preparations. Arch Ophthalmol 1988;106:484-7.

5. Polack FM, McNiece MT. The treatment of dry eyes with Na hyaluronate (Healon): a preliminary report. Cornea 1982;1:133-6.

6. Stuart JC, Linn JG. Dilute sodium hyaluronate (Healon) in the treatment of ocular surface disorders. Ann Ophthalmol 1985;17:190-2.

7. DeLuise VP, Peterson WS. The use of topical Healon tears in the management of refractory dry-eye syndrome. Ann Ophthalmol 1984;16:823-4.

8. Aragona P, Papa V. Micali A, et al. Long term treatment with sodium hyaluronatecontaining artificial tears reduces ocular surface damage in patients with dry eye. Br J Ophthalmol 2002;86:181-4.

9. Limberg MB, McCaa C, Kissling GE, et al. Topical application of hyaluronic acid and chondroitin sulfate in the treatment of dry eyes. Am J Ophthalmol 1987;103:194-7.

10. Shimmura $\mathbf{S}$, Ono M, Shinozaki K, et al. Sodium hyaluronate eye drops in the treatment of dry eyes. Br J Ophthalmol 1995;70:1007-11.

11. Condon PI, McEwen CG, Wright M, et al. Double blind, randomized, placebo controlled, crossover, multicentre study to determine the efficacy of a $0.1 \%(\mathrm{w} / \mathrm{v})$ sodium hyaluronate solution (Fermavisc) in the treatment of dry eye syndrome. Br J Ophthalmol 1999;83:1121-4.

12. Nakamura S, Okada S, Umeda Y, et al. Development of a rabbit model of tear film instability and evaluation of viscosity of artificial tear preparations. Cornea 2004;23:390-7.

13. Nakamura M. Hikida M, Nakano $\mathrm{T}$, et al. Characterization of water retentive properties of hyaluronan. Cornea 1993;12:433-6.

14. Nishida T, Nakamura M, Mishima $\mathrm{H}$, et al. Hyaluronan stimulates corneal epithelial migration. Exp Eye Res 1991;53:753-8.

15. Sugiyama T, Miyauchi S, Machida A, et al. The effect of sodium hyaluronate on the migration of rabbit corneal epithelium. The effect of topical administration. J Ocular Pharmacol 1991;7:53-64. 
16. Gomes JAP, Amankwah R, Powell-Richards A, et al. Sodium hyaluronate promotes migration of human corneal epithelial cells in vitro. Br J Ophthalmol 2004;88:821-5.

17. Watanabe T, Kawashima M, Kawai M, et al. Effect of sodium hyaluronate eyedrops on tear meniscus height determined by fluorescein photography. [in Japanese] Folia Ophthalmol Jpn 2004;55:369-73.

18. Tsubota K, Yamada M. Tear evaporation from the ocular surface. Invest Ophthalmol Vis Sci 1992;33:2942-50.

19. Mengher LS, Pandher KS, Bron AJ, et al. The effect of sodium hyaluronate $(0.1 \%)$ on break-up time (NIBUT) in patients with dry eyes. Br J Ophthalmol 1986;70:442-7.

20. Hamano T, Horimoto $\mathrm{K}$, Lee $\mathrm{M}$, et al. Evaluation of the effect of sodium hyaluronate ophthalmic solution on tear film stability. [in Japanese] J Jpn Ophthalmol Soc 1993;97:928-32.
21. Johnson ME, Murphy PJ, Boulton M. Effectiveness of sodium hyaluronate eyedrops in the treatment of dry eye. Graefe's Arch Clin Exp Ophthalmol 2006;244:109-12.

22. Snibson GR, Greaves JL, Soper NDW, et al. Precorneal residence times of sodium hyaluronate solutions studied by quantitative gamma scintigraphy. Eye 1990;4:594602.

23. Gurny R, Ryser JE, Tabatabay C, et al. Precorneal residence time in humans of sodium hyaluronate as measured by gamma scintigraphy. Graefe's Arch Clin Exp Ophthalmol 1990;228:510-2

24. Snibson GR, Greaves JL, Soper NDW, et al. Ocular surface residence times of artificial tear solutions. Cornea 1992;11:288-93.

25. Mishima S, Gasset A, Klyce Jr SD, et al. Determination of tear volume and tear flow. Invest Ophthalmol Vis Sci 1966;5:264-76.

26. Asari A, Morita M, Sekiguchi T, et al. Hyaluronan, CD44 and fibronectin in rabbit corneal wound healing. Jpn J Ophthalmol 1996;40:18-25.

\section{Keep up to date: sign up for our alerting services}

Find out automatically when an article is published on a specific topic or by a particular author. We can also alert you when an article is cited or if an eLetter or correction is published. You can also choose to be alerted when a new issue is published online [and when we post articles Online First]. Check out the New Content Alerts and Citation tracker from the Online tools section on the home page. 